\title{
EDITORIAL
}

\section{Opportunities in open data}

The open source concept emerged as a way to describe the idea that, open and shared access to the code of a program, allows to improve it and consequently, to evolve. This way of collaboration has grown until it became a culture that currently penetrates other scenarios of society, as it is the Open Government. This concept corresponds to the influence that the seeking of "common wealth" has upon institutions and their governors. The same way these are related to the technological, economical and human development of societies.

Open data are records that can be freely used, re-used and redistributed by any person. They are subject only, at most, to the requirement to attribute and/or share-alike. In this editorial, we give a brief look at the use of open data, coming from different sources, contribute to the analysis of events, for example, territorial events with the aim of building, among others, indicators that help solve problems of urban planning, socio-spatial segregation, territorial poverty and the effectiveness of political policies in general.

Data coming from ministries, intendancies, governorates, municipalities, universities and schools, among others, are associated with a wide variety of "public" information (shared ownership capital) that might interest different users. These records enjoy, mostly, of statistical reliability, by providing methodological documents of collection. This quality is highly appreciated, due to the fact that in economy as in other fields, the scarcity or inexistence of statistics data at a micro-territorial level are, typically addressed, with techniques of prediction and/or disaggregation.

In our opinion, the importance of open data radicates in a group of reasons, described by the Open Knowledge Foundation, and that bonds this concept to the open government:

1. Transparency. In a well-functioning, democratic society citizens need to know what their government is doing. To do that, they must be able freely to access government data and information and to share that information with other citizens.

2. Releasing social and commercial value. In a digital age, data is a key resource for social and commercial activities. By opening up data, government can help drive the creation of innovative business and services that deliver social and commercial value.

3. Participatory Governance. By opening up data, citizens are enabled to be much more directly informed and involved in decision-making. This is more than transparency: it's about making a full "read/write" society, not just about knowing what is happening in the process of governance but being able to contribute to it.

From the year 2009, Chile has a specific law to regulate the access to public information, since it became a member of the Open Government Partnership. This international organization requires member countries to develop policies in the priority areas: promotion of standards of professional integrity, increase in the access to the new technologies for the opening and account declaration, the access to information about the gubernatorial activities and citizen participation support through transparency about policies formulation and decision-making. As an example, the access to information 
of government related activities can be made in the datos.gob.cl platform, which contains published data from different public institutions under a standard scheme which facilitates a compared analysis. In this same form, the Library of National Congress also distributes its information in the web site datos.bcn.cl.

The development of Data Engineering, Business Intelligence and Geographic Information Systems (GIS) solutions has allowed the users to obtain new information, different to the one initially set by those who provide the data. This was achieved through crossing, classification, learning or integration of data from different sources. In this sense, our team has covered a work line that integrates technologies and open data to the following applications:

- From the data evolution, contrasting information and analyzing, for example, the effects that public policies have produced in a certain population, throughout time.

In this sense, we have worked on the integration of information gathered in the National Census of people, houses and homes in Chile since 1992. For that, we have been supported by the use of Database and ETL (Extraction, Transformation, and Loading) technology.

- In second place, it is interesting to situate this data spatially, which implies to observe, how the changes in the environments have affected a population, and how these changes in this population have affected the environment.

In this way, we have worked in situating spatially the information of the Census, using essentially, Spatial Database, SIG and Web Map Server technology.

- Finally, we propose that integration of data coming from different sources would produce an effect of synergy, allowing us to see aspects that were hidden to the individual sight.

In this area, we have worked in integrating the information of the Census in Chile, with the CASEN (National Socio-Economic Characterization Survey). In this process, we used an extrapolation technique called Spatial Matching, which searches one or more observations in the Census, that are statistically similar to extrapolate the data in a micro territorial unit that is unavailable in the survey. The developed solution has incorporated ETL, Data warehouse, OLAP and Dashboards technologies.

In the future, the use of technologies, like Data Mining, Computational Intelligence and others alike, it is also considered as potential possibilities.

As we stated, the opportunities exist. Analyzing open data of different sources, especially governmental related ones, is feasible. It only depends on its efficient use.

\section{Ricardo Valdivia}

Escuela Universitaria de Ingeniería Industrial, Informática y Sistemas Universidad de Tarapacá Arica-Chile rvaldivi@uta.cl
Mónica Navarrete

Escuela Universitaria de

Administración y Negocios Centro de Estudios Regionales (CEUTA) Universidad de Tarapacá Arica-Chile mnavarre@uta.cl

\section{Diego Aracena}

Escuela Universitaria de Ingeniería Industrial, Informática y Sistemas Universidad de Tarapacá Arica-Chile daracena@uta.cl 